\title{
Increasing Polymer Solar Cell Efficiency with Triangular Silver Gratings
}

\author{
Aimi Abass, Honghui Shen, Peter Bienstman, Bjorn Maes \\ Photonics Research Group, Ghent University - imec, Department of Information Technology, St.-Pietersnieuwstraat 41, 9000 Ghent, \\ Belgium \\ aimi.abass@ugent.be
}

\begin{abstract}
We investigate strongly enhanced light absorption in a thin P3HT:PCBM solar cell with a triangular silver grating back contact. The correlation between grating and plasmonic absorption spectrum features are identified and studied with rigorous numerics.
\end{abstract}

(C)2010 Optical Society of America

OCIS codes: (040.5350) Photovoltaic; (240.6680) Surface plasmons

\section{Introduction}

The control of plasmonic mode excitation by incoming light via periodic metallic gratings was shown to be a powerful tool for increasing solar cell absorption. In some cases, the gratings are present in order to avoid the excitation of these modes and to increase the reflection from the metallic back contact, e.g. for fairly thick inorganic solar cells [1]. In other cases, the gratings allow to actually utilize the plasmonic modes, e.g. for thin film polymer solar cells with a thickness on the order of 100nm [2]. Recently, our group studied the increase of absorption efficiency by incorporating silver nanoparticles inside a thin polymer solar cell [3]. These works show that the subwavelength confinement of plasmonic modes can help for organic solar cell enhancement, as thin cells are required in order to maintain an efficient charge collection.

Here we numerically study the performance of a triangular silver grating with 1D periodicity, as a back contact of a P3HT:PCBM bulk heterojunction solar cell. A geometrically similar solar cell structure with different metal and polymers was presented earlier in [2] without an in-depth analysis of the structural effects, which we offer here. For the active layer, we determine an integrated absorption efficiency increase of $15.6 \%$ throughout the 300-800nm spectral region (weighted with the AM1.5G spectrum). This is mainly achieved by extending the absorption edge (around 600nm for P3HT:PCBM) to significantly higher wavelengths.

We consider an infinitely periodic grating structure, excited with a plane wave, as depicted in Fig. 1(a). The rigorous simulations were performed in 2D using the frequency domain finite element software COMSOL. We studied the system for a wide range of parameters: polymer thickness, grating period, fill factor and height. Here we focus on results for an active layer of 150nm thickness.

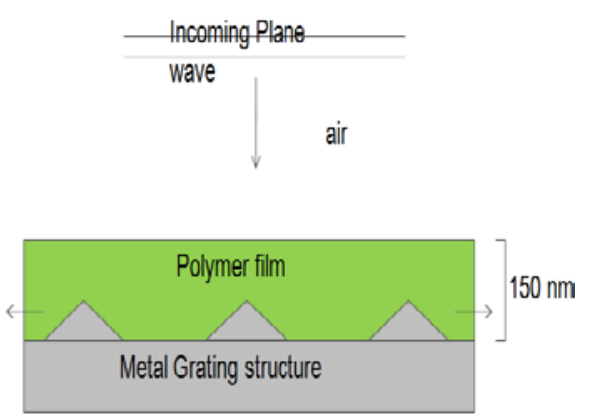

(a)

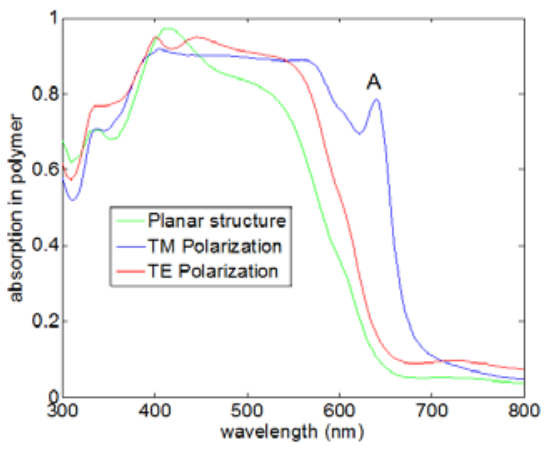

(b)

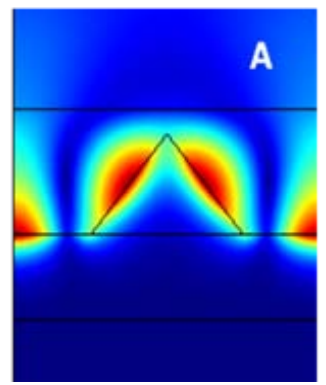

(c)

Fig. 1. (a) The simulated system, a plane wave incoming at normal incidence to the solar cell structure. (b) Polymer absorption spectrum for the planar and patterned device, respectively. The patterned device has a period of $400 \mathrm{~nm}$ with fill factor 0.5 and triangle height $120 \mathrm{~nm}$. (c) Plot of the norm of the magnetic field profile for the TM polarization input at 640nm wavelength.

\section{Results}

Figure 1(b) shows an example of enhancement in the absorption spectrum that can be achieved by the introduction of a triangular grating (grating periodicity $400 \mathrm{~nm}$, fill factor 0.5 and triangle height $120 \mathrm{~nm}$ ). The peak at $640 \mathrm{~nm}$ (for TM) is due to excitation of a plasmonic mode. The plot of the field profile at this wavelength is shown in figure 1(c). The field is distributed mostly in the polymer, leading to a huge enhancement at this wavelength. The integrated absorption efficiency for a planar unpatterned structure is $48 \%$ while for a patterned structure it is $63.6 \%$ for TM polarization and $56 \%$ for the TE Polarization. The absorption enhancement for the TE polarization is due to scattering by the grating profile. The geometric parameters of the grating were optimized to obtain a large integrated absorption efficiency, but also to have a significant enhancement at the 
absorption edge of the active layer. Therefore we aimed for resonances in the wavelength range of 600 to $650 \mathrm{~nm}$. A recent publication indicated that the internal quantum efficiency of P3HT:PCBM is still around 70\% in this range [4].

As already mentioned, the peak appearing in figure 1(b) stems from a surface plasmon effect. More specifically, it comes from a Surface Plasmon Polariton (SPP) mode associated with a flat polymer-silver interface. We see this by examining the 'folding' of the dispersion relation of the SPP mode induced by the 400nm periodicity, which is shown in figure 2(a). We are interested in the crossings at $\mathrm{k}=0$ because this corresponds to modes that can be excited by perpendicularly incident waves. Besides imposing periodicity, the grating will induce a perturbation and thus split the $\mathrm{k}=0$ degenerate modes in figure 2(a). As we make the grating more pronounced by increasing the height or fill factor, the splitting of the crossings becomes larger.

Figure 2(b) shows a plot of the integrated efficiency as a function of grating fill factor and triangle height at 400nm period. As indicated in the figure, there is an optimum combination for the fill factor and height which is due to the complex interaction of the SPP peak position and strength, and the grating scattering capability.

Figure 2(c) shows the absorption spectrum at different points in figure 2(b). We see in figure 2(c), as we go from graph A, B, to C (corresponding to triangle heights10nm, $60 \mathrm{~nm}$, and $120 \mathrm{~nm}$ ), that the peak position stems from the flat SPP mode at 800nm which gets blue shifted. Indeed, as the triangle height increases, a stronger perturbation splitting effect is observed.

Following graph C, D and E in figure 2(c), we follow the peak position when we change the fill factor of the grating. When we decrease the fill factor (similar to decreasing the grating height) the perturbation is weaker. Therefore we see a red shift of the peak from $640 \mathrm{~nm}$ (C) to $697.5 \mathrm{~nm}$ (D). From C to E we see the opposite. The peaks related to the second crossing near $450 \mathrm{~nm}$ in figure 2(a) are not seen because there the polymer absorption is high.

As we increase the height, the scattering capability of the triangular grating profile increases. This fact is seen from the absorption enhancement in the wavelength range where there is no plasmonic mode excitation. For example, as we again progress from point A to $C$ in figure 2(c), we see that there is overall enhancement in the 500-600nm region which can only be attributed to increased scattering.
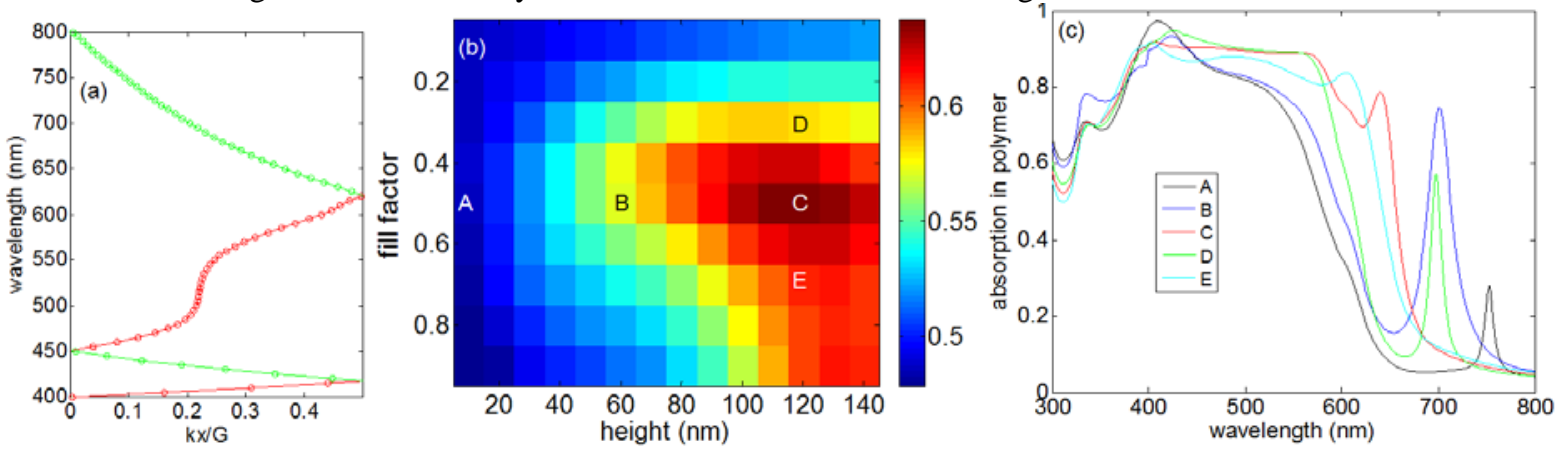

Fig. 2. (a) Dispersion diagram of SPP mode at a flat polymer-silver interface folded with 400nm grating period, where G is the reciprocal lattice vector. (b) Dependence of integrated absorption efficiency in the 300-800nm region . (c) TM polarization absorption spectrum at different points in (b).

\section{Conclusions}

We performed detailed numerical studies of the potential of 1D triangular silver gratings as a back contact for P3HT:PCBM bulk heterojunction solar cells to increase the absorption efficiency. We show a $15.6 \%$ increase of integrated absorption efficiency in the 300-800nm wavelength range. Moreover, most of the increase of efficiency occurs in the region for which the internal quantum efficiency can still be optimal. The correspondence between the plasmon peak positions and the grating geometric parameters was studied.

We acknowledge B. Niesen and support from IWT-SBO No.060843 “Polyspec”, grant No. IAP P6-10 “photonics@be” and COST MP0702.

\section{References}

[1] F.-J. Haug, T. Söderström, O. Cubero, V. Terrazzoni-Daudrix, and C. Ballif, "Plasmonic absorption in textured silver back reflectors of thin film solar cells", Journal of Applied Physics, 104, p064509 (2008).

[2] K. Tvingstedt, N. Persson, O. Inganäs, A. Rahachou and I.V. Zozoulenko, "Surface plasmon increase absorption in polymer photovoltaic cells”, Appl. Phys. Lett., 91, p113514 (2007).

[3] H. Shen, P. Bienstman, and B. Maes, "Plasmonic absorption enhancement in organic solar cells with thin active layers", Journal of Applied Physics, 106, p073109 (2009).

[4] G.F. Burkhard, E.T. Hoke, S.R. Scully, and M.D. McGehee, "Incomplete exciton harvesting from fullerenes in bulk heterojunction solar cells”, Nano Letters, 9, p4037 (2009). 